\title{
Uneven intervertebral motion sharing is related to disc degeneration and is greater in patients with chronic, non-specific low back pain: an in vivo, cross-sectional cohort comparison of intervertebral dynamics using quantitative fluoroscopy
}

\author{
Alan Breen $^{1}$ (D) Alexander Breen ${ }^{2}$ \\ Received: 17 November 2016/Revised: 3 April 2017/ Accepted: 24 May 2017/Published online: 29 May 2017 \\ (c) The Author(s) 2017. This article is an open access publication
}

\begin{abstract}
Purpose Evidence of intervertebral mechanical markers in chronic, non-specific low back pain (CNSLBP) is lacking. This research used dynamic fluoroscopic studies to compare intervertebral angular motion sharing inequality and variability (MSI and MSV) during continuous lumbar motion in CNSLBP patients and controls. Passive recumbent and active standing protocols were used and the relationships of these variables to age and disc degeneration were assessed.

Methods Twenty patients with CNSLBP and 20 matched controls received quantitative fluoroscopic lumbar spine examinations using a standardised protocol for data collection and image analysis. Composite disc degeneration (CDD) scores comprising the sum of Kellgren and Lawrence grades from L2-S1 were obtained. Indices of intervertebral motion sharing inequality (MSI) and variability (MSV) were derived and expressed in units of proportion of lumbar range of motion from outward and return motion sequences during lying (passive) and standing (active) lumbar bending and compared between patients and controls. Relationships between MSI, MSV, age and CDD were assessed by linear correlation.
\end{abstract}

Electronic supplementary material The online version of this article (doi:10.1007/s00586-017-5155-y) contains supplementary material, which is available to authorized users.

Alan Breen

abreen4@bournemouth.ac.uk

1 Faculty of Science and Technology, Bournemouth University, Poole BH12 5BB, UK

2 Institute for Musculoskeletal Research and Clinical Implementation, Anglo-European College of Chiropractic, Bournemouth BH5 2DF, UK
Results MSI was significantly greater in the patients throughout the intervertebral motion sequences of recumbent flexion $(0.29$ vs. $0.22, \mathrm{p}=0.02)$ and when flexion, extension, left and right motion were combined to give a composite measure (1.40 vs. $0.92, p=0.04)$. MSI correlated substantially with age $(R=0.85, p=0.004)$ and CDD $(R=0.70, p=0.03)$ in lying passive investigations in patients and not in controls. There were also substantial correlations between MSV and age $(R=0.77, p=0.01)$ and CDD $(R=0.85, p=0.004)$ in standing flexion in patients and not in controls.

Conclusion Greater inequality and variability of motion sharing was found in patients with CNSLBP than in controls, confirming previous studies and suggesting a biomechanical marker for the disorder at intervertebral level. The relationship between disc degeneration and MSI was augmented in patients, but not in controls during passive motion and similarly for MSV during active motion, suggesting links between in vivo disc mechanics and pain generation.

Keywords Back pain - Spinal injuries · Kinematics · Fluoroscopy · Diagnosis

\section{Background}

Concepts of lumbar spine stability cover a range of complexity. Intervertebral angle change (IV-RoM) is not now thought to be very useful, due to wide population variations, although range of translation is generally preferred by spinal surgeons who assess for instability [1]. These measures, although of questionable validity, are nevertheless accessible from plain radiographs. To probe more deeply and investigate more subtle forms of instability, 
continuous intervertebral motion measures are needed [2-5].

For the assessment of mechanical destabilisation of the spine caused by injury, the intervertebral neutral zone is thought to be the most sensitive measure [6]. Although its measurement has been generally confined to cadaveric studies, the advancing sophistication of quantitative fluoroscopic systems (QF) is beginning to provide a surrogate in vivo measure in the form of slope of the intervertebral rotation-time curve (also known as the attainment rate) [7-10].

Chronic, non-specific low back pain (CNSLBP) is widely considered to be at least partially of mechanical origin, due to its susceptibility to movement and position. It is also considered to be related to intervertebral disc degeneration [11]. However, no reliable diagnostic tool that could help a clinician to determine if a disc is the source of the pain in patients with chronic LBP is currently available [12]. Instead, relationships between trunk myoelectric activity, co-ordination and directional preference are more prevalent in the clinical biomechanics literature [13-15]. However, without an assessment of the relationship between segmental mechanics and pain, identification of biomechanical markers in CNSLBP will remain elusive.

While the in vivo investigation of intervertebral loading is still problematical, kinematic studies are becoming more common [16]. The lumbar spine is a kinetic chain that requires the sharing of motion between levels during bending. Various aspects of intervertebral motion sharing have been investigated in cadaveric studies [17-19] in plain radiographic studies in vivo [20-24] and in continuous radiographic studies [3, 5, 8, 20, 25-30]. Most of these have studied motion onset and displacement; however, two that studied displacement [4, 24] and one that studied pattern variations [29], found differences between patients with CNSLBP and controls.

Intervertebral motion pattern variation studies are of interest because they provide more information than end of range studies and can be more readily applied to contemporary concepts of spine stability. Reeves and Cholewicki [31] identified impaired restraint and performance in the passive and active intervertebral subsystems as subset measures of subtle instability, where restraint is the ability of a system to resist an imposed perturbation and performance is the ability to return to the original position once the perturbation has been removed. In the lumbar spine, the average range of differences in the sharing of motion by each intervertebral level over the sequence reflects the inequality of restraint across levels (MSI) (Fig. 1). Its variability throughout the motion (MSV), may be considered as an expression of intervertebral motion control.

In assessing the possible role of intervertebral motion sharing in CNSLBP, Mellor et al. [29] investigated the variability of recumbent intervertebral passive motion sharing and found it to be greater in patients with CNSLBP than in controls. Therefore, it was thought as a possible biomechanical marker for CNSLBP. These studies only addressed motion sharing variability (control) and not equality of restraint-and their replication has not been attempted until now. They also did not account for the effects of disc degeneration. We, therefore, attempted to replicate these studies, adding a measure of motion sharing inequality, investigating motion under load and incorporating disc degeneration as a possible source of interaction with these measures.

\section{Objectives}

1. To see if previous investigations of differences between patients with CNSLBP and healthy controls using passive recumbent motion could be replicated as a biomechanical marker [29].

2. To determine if these differences were also present during standing flexion motion investigations.

3. To determine the relationships between uneven motion sharing and age and disc degeneration.

\section{Methods}

We conducted two cross-sectional, prospective observational studies of intervertebral motion sharing in the lumbar spine - one during passive recumbent motion and the other during active weight bearing motion.

\section{Participants}

Forty participants were recruited. Twenty were patients who had been referred for continuous radiographic (QF) studies for CNSLBP and 20 were healthy control volunteers recruited from staff, students and visitors of our institution. Controls were matched as closely as possible to patients for age and gender. Participants were divided into two cohorts. Cohort 1 had received passive, recumbent QF investigations in left, right, flexion and extension motion similar to the 2014 study of Mellor [29] and Cohort 2 had received active standing flexion QF investigations only. Participants could be included if they were: male or female, age $21-80$, BMI $<30$, with no history of previous back or abdominal surgery or spondylolisthesis, no medical radiation exposure of $>8 \mathrm{mSV}$ in the previous 2 years and no pregnancy (females). Controls had to have been free of any back pain that limited their normal activity for more than 1 day in the previous year and patients had to have had 


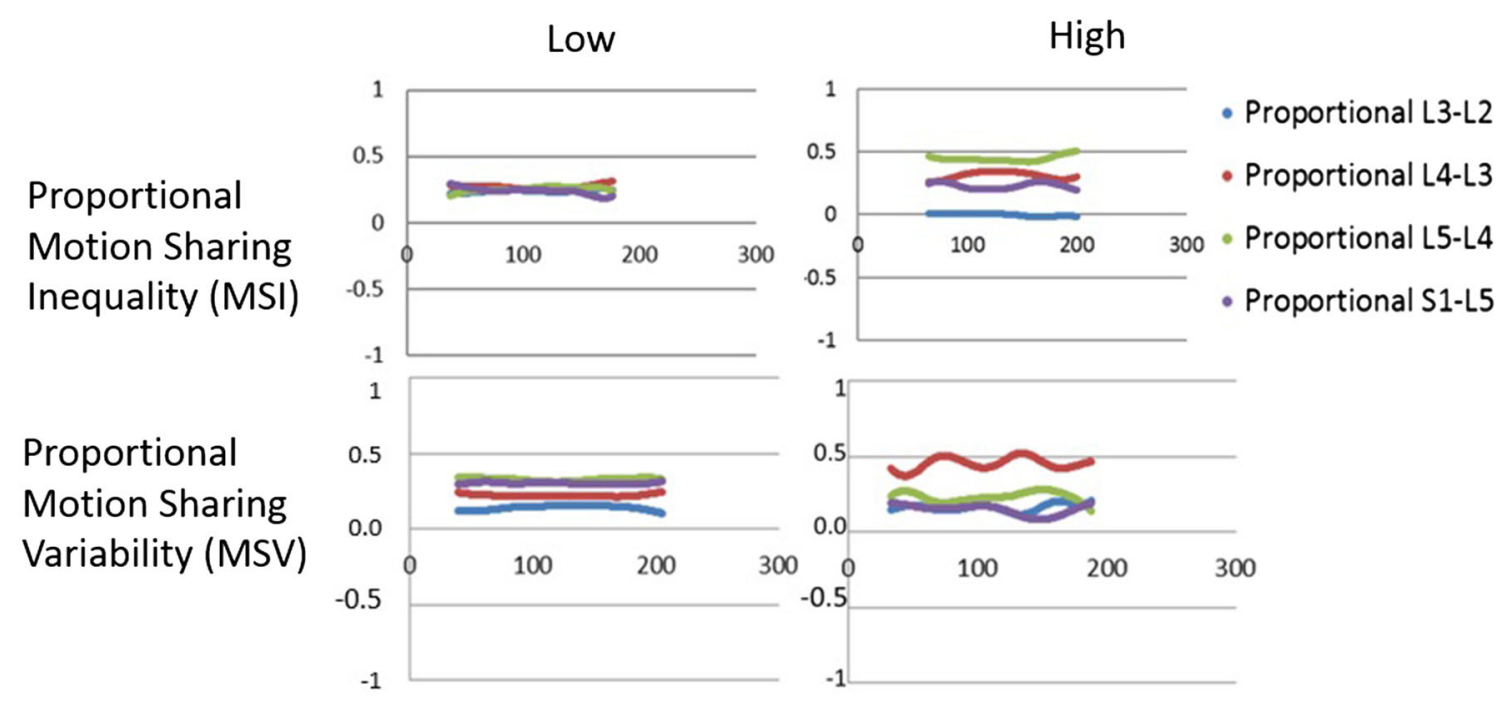

Fig. 1 Examples of equal, unequal, regular and variable continuous proportional motion sharing

their back pain for longer than 3 months. All participants gave informed consent. The study received a favourable ethical opinion by the National Research Ethics Service (South West 3, REC reference 10/H0106/65).

\section{Image acquisition and analysis}

QF image acquisition and analysis of Cohort 1 were similar to that described by Mellor et al. [29]. Briefly, participants lay on a movable table in which the trunk section was motorised and driven by a controller (Atlas Clinical Ltd.), which caused it to execute a bending angle of $40^{\circ}$ during separate left, right, flexion and extension motion sequences while fluoroscopic screening took place. For Cohort 2, participants stood with their right side against an upright motion frame with their pelvises secured and their arms on a projecting rest which guided them through a flexion angle of $60^{\circ}$ and back using the same controller apparatus as for the lying procedure. Thus, Cohort 1 received passive, recumbent motion in two planes and four directions and Cohort 2, active, weight bearing motion in flexion only.

The motion controllers accelerate at $6^{\circ} \mathrm{s}^{-2}$ for the first second followed by a uniform $6^{\circ} \mathrm{s}^{-1}$ thereafter. The images were collected at $15 \mathrm{~Hz}$ using a Siemens Arcadis Avantic digital C-arm fluoroscope (Siemens GMBH). Images were exported to a computer workstation and analysed using manual first image registration and thereafter bespoke frame-to-frame tracking using codes written in Matlab (V2013-the Mathworks Inc). Anonymised image sequences were analysed by one operator $(\mathrm{AxB})$ and outputted to an Excel spreadsheet in the form of frame-toframe measurements of intervertebral angular rotation throughout each motion sequence. Accuracy and repeatability for intervertebral rotations using this method have been determined as: accuracy (side bending $0.32^{\circ}$, flexionextension $0.53^{\circ}$ ) [32]. Inter and intra observer repeatability for left, right, flexion and extension recumbent motion ranged from ICC $0.74-0.99$ and SEM $0.08^{\circ}-0.77^{\circ}$ and for weight bearing flexion from ICC $0.94-0.96$, SEM $0.23^{\circ}-$ $0.61^{\circ}[9,29]$. The rotational angles at each intervertebral level were transformed by a second operator $(\mathrm{AB})$ into proportional motion of the segments of lumbar spine (L2L5 in passive recumbent motion and L2-S1 in standing flexion). The proportional motion of segments refers to their relative contributions to the motion of the lumbar spine at all points in the bend [29].

Two parameters of the proportional motion sharing were extracted: motion sharing inequality (MSI) and motion sharing variability (MSV) (Fig. 2). A full account of the

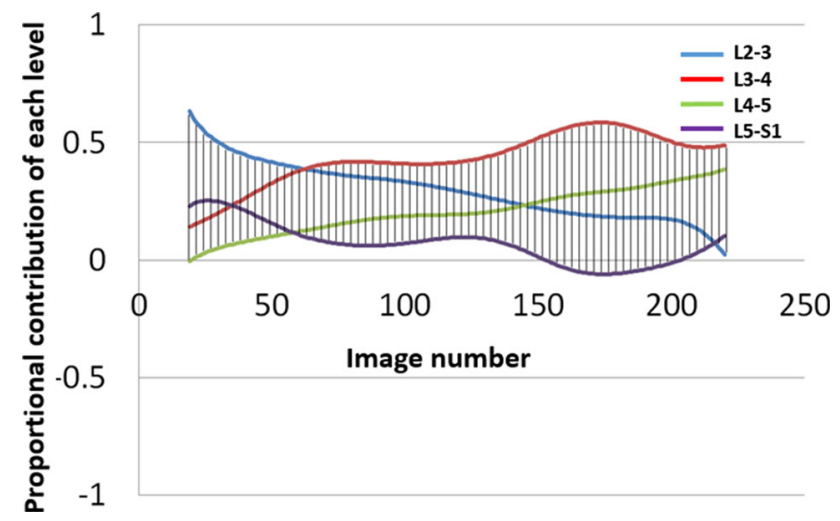

Fig. 2 Derivation of MSI and MSV. Example of the measurement of continuous proportional intervertebral range of motion (flexion). The range was calculated for each data point on the $x$-axis (black lines). Motion share inequality (MSI) was calculated as the mean of all the ranges in the sequence and motion share variability (MSV) was their RMS value (see also Electronic Supplementary Material) 
derivation is given in the Online Appendix, but briefly, MSI was calculated as the average filtered range contributions to the motion ( $\mathrm{fRC}_{\mathrm{i}}$ ) across the $N$ image data points remaining after filtering (see Online Appendix).

$\mathrm{MSI}=\frac{\sum_{i=1}^{N} \mathrm{fRC}_{i}}{N}$.

MSV was calculated as the square root of the variance of these distances across all data points in each sequence:

$\mathrm{MSV}=\sqrt{\frac{\sum_{i=1}^{N}\left(\mathrm{fRC}_{i}-\mathrm{MSI}\right)^{2}}{N}}$.

Both measures were expressed in units of proportion, with MSI being the unevenness in restraint between segments and MSV the unevenness of control.

The initial lateral projection images of all sequences were assessed for disc degeneration using the Kellgren and Lawrence rating scale by a chiropractor $(\mathrm{AB})$ who was trained to interpret radiographs, giving a score of $0-4$ for each level [33]. These were added together to give a composite disc degeneration score (CDD) for each lumbar spine. The sum of disc degeneration scores was used in an effort to accommodate both a single point of injury and regional lumbar dysfunction as pain generators. The same images were assessed by the same operator 1 month later to assess reliability.

\section{Statistical analysis}

All data were inspected for normality using the ShapiroWilk test. Averages of non-normal data were expressed as medians and the significance of their differences and correlations calculated using the two-way Mann-Whitney and Spearman rank correlation tests. Averages of normal data were expressed as means and their differences and correlations were examined using two-way unpaired $t$ tests and Pearson's $R$ for correlations. The significance of differences in proportions was calculated using the Fisher exact test.
For the 20 participants (Cohort 1) who received recumbent flexion, extension, left and right imaging, the MSI and MSV values were summed, as in Mellor et al. [29], whereas for Cohort 2, the values for weight bearing flexion were calculated for only one plane of motion. For comparison, MSI and MSV for recumbent flexion in Cohort 1 were also calculated and compared.

\section{Results}

Image sequences of 83 referred patients were drawn from a group of patients with CNSLBP who had been referred for QF investigations (Fig. 3). For 14 of these, referrers had requested recumbent flexion, extension, left and right examinations and in 12 , weight bearing flexion. Four patients were excluded from Cohort 1 and 2 from Cohort 2 due to spondylolisthesis or previous spinal surgery.

The characteristics of patients and controls $(n=40)$ are shown in Table 1. Age ranges were wide, accommodating a degree of difference in disc degeneration grades, which nevertheless averaged in the lower third of the possible range. At the second assessment for disc degeneration grade 1 month later, one disc's grade was revised from 1 to 0 and one from 0 to 1 . There were no significant differences between patients and controls in terms of age, sex or combined disc degeneration grade.

Both combined and flexion only MSIs were significantly higher in the patients than the controls in Cohort 1 (combined MSI in patients: 1.40, controls 0.92, $p=0.04$; flexion MSI in patients 0.29 , controls $0.22, p=0.02$ ), but there were no significant differences in MSV, as found by Mellor et al. [29] (Table 2).

Correlations between age, combined disc degeneration (CDD), MSI and MSV are shown for each cohort examination in Table 3 and scatter plots on which these correlations are based are presented in Fig. 3a-h.

Fig. 3 Patient populations

CNSLBP patients referred for QF

$\mathrm{N}=83$

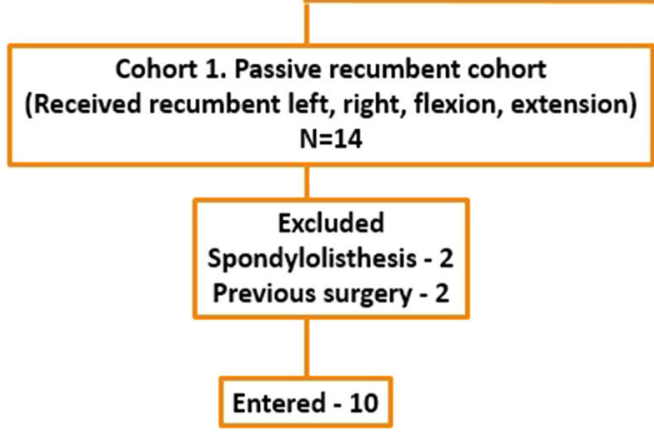

Cohort 2. Flexion only cohort (Received weight bearing flexion) $\mathrm{N}=12$

Excluded Spondylolisthesis - 1 Previous surgery - 1

Entered - 10 
Table 1 Characteristics of patients and controls $(n=40)$

\begin{tabular}{llll}
\hline & Patients & Controls & $p$ \\
\hline Cohort 1 & & & \\
$N$ & 10 & 10 & \\
Age (mean, SD) & $51(14.9)$ & $49(12.3)$ & 0.83 \\
Sex & M 7, F 3 & M 8, F 2 & 0.65 \\
DD/16 (mean, SD) & 4.7 (3.0) & $4.4(3.1)$ & 0.22 \\
Cohort 2 & & & \\
$N$ & 10 & 10 & \\
Age (mean, SD) & $49(13.0)$ & $47(14.2)$ & 0.78 \\
Sex & M 6, F 4 & M 6, F 4 & 0.99 \\
DD/16 (median, range) & $5.0(2-10)$ & $3.0(1-10)$ & 0.22 \\
\hline
\end{tabular}

Table 2 Comparison of patients and controls by MSI and MSV

\begin{tabular}{|c|c|c|c|}
\hline & Patients $(n=10)$ & Controls $(n=10)$ & $p$ \\
\hline \multicolumn{4}{|c|}{ Cohort 1 (lying flexion + extension + left + right $)$} \\
\hline MSI (mean) & 1.48 & 0.92 & 0.04 \\
\hline MSV (median) & 0.19 & 0.15 & 0.25 \\
\hline \multicolumn{4}{|c|}{ Cohort 1 (lying flexion) } \\
\hline MSI (median) & 0.29 & 0.22 & $\mathbf{0 . 0 2}$ \\
\hline MSV (mean) & 0.08 & 0.08 & 0.63 \\
\hline \multicolumn{4}{|c|}{ Cohort 2 (standing flexion) } \\
\hline MSI (mean) & 0.39 & 0.33 & 0.25 \\
\hline MSV (median) & 0.08 & 0.14 & 0.97 \\
\hline
\end{tabular}

Significant correlations $(p<0.05)$ shown in bold

There were substantial correlations between age, disc degeneration and MSI in combined passive recumbent motion in the Cohort 1 patients, but not in controls (Fig. 4a-d). In weight bearing active flexion motion in the Cohort 2 patients, there were also substantial correlations between age, disc degeneration and uneven motion, exhibited as variability of motion sharing (MSV) (Fig. 4eh). This was also exclusive to patients with CNSLBP. (MSI appears to have been unrelated.) There was moderate correlation between MSI and MSV in both cohorts, which only reached significance in controls, although it was present in all groups except combined recumbent motion.

\section{Discussion}

In recent years, the usefulness of dynamic analysis of spinal disorders has become more apparent. For example, Shiba et al. [34] found that by examining dynamic, as opposed to static global sagittal alignment at the beginning and end of a gait sequence in patients with degenerative lumbar kyphoscoliosis, loss of global sagittal alignment at the end of the gait sequence was more readily detected.

The present studies bring to three the number of cohorts in which uneven continuous motion sharing has been found to be greater in CNSLBP patients than in controls. In the present study, uneven passive restraint across the lumbar spine (MSI) was greater in patients with CNSLBP than in pain free controls. There would seem to be at least two possible explanations for this. The first is that unequal restraint (MSI) could add to increased muscle metabolic demands during activities of daily living causing recurrent muscle pain [35]. The second may relate to the rapid accelerations associated with inadequate restraint at an injured level, which has been suggested to be a nociceptive pain generator producing a single point of pain. This could also cause motion sharing inequality [36, 37].

It is notable that age and disc degeneration were substantially correlated with uneven passive motion sharing (MSI) in the back pain patients and hardly at all in the controls in Cohort 1. This suggests that CNSLBP is linked

Table 3 Correlations between age, combined disc degeneration, motion share inequality and motion share variability in patients and controls $(n=40)$

\begin{tabular}{|c|c|c|c|c|c|c|c|c|c|c|c|c|c|}
\hline & & \multicolumn{6}{|c|}{ Patients } & \multicolumn{6}{|c|}{ Controls } \\
\hline & & \multicolumn{2}{|l|}{ CDD } & \multicolumn{2}{|l|}{ MSI } & \multicolumn{2}{|l|}{ MSV } & \multicolumn{2}{|l|}{ CDD } & \multicolumn{2}{|l|}{ MSI } & \multicolumn{2}{|c|}{ MSV } \\
\hline & & $R$ & $p$ & $R$ & $p$ & $R$ & $p$ & $R$ & $p$ & $R$ & $p$ & $R$ & $p$ \\
\hline Cohort 1 recumbent & Age & 0.94 & 0.0003 & 0.85 & 0.004 & 0.11 & 0.68 & 0.76 & 0.015 & 0.12 & 0.76 & 0.2 & 0.58 \\
\hline Flx + Ext + left + right & CDD & & & 0.70 & $\mathbf{0 . 0 3}$ & -0.21 & 0.54 & & & -0.15 & 0.67 & 0.07 & 0.85 \\
\hline L2-5 (Spearman) & MSI & & & & & 0.01 & 0.97 & & & & & 0.77 & 0.01 \\
\hline Cohort 1 recumbent & Age & 0.94 & 0.0003 & 0.27 & 0.58 & -0.19 & 0.58 & 0.76 & 0.015 & 0.33 & 0.34 & 0.27 & 0.48 \\
\hline Flexion only & CDD & & & 0.58 & 0.28 & 0.01 & 0.99 & & & 0.13 & 0.73 & 0.09 & 0.81 \\
\hline L2-5 (Spearman) & MSI & & & & & 0.44 & 0.2 & & & & & 0.27 & 0.45 \\
\hline Cohort 2 weight bearing & Age & 0.83 & 0.005 & 0.54 & 0.11 & 0.77 & 0.01 & 0.68 & 0.039 & 0.26 & 0.24 & 0.25 & 0.49 \\
\hline Flexion only & CDD & & & 0.43 & 0.23 & 0.85 & 0.004 & & & 0.39 & 0.26 & 0.47 & 0.18 \\
\hline L2-S1 (Spearman) & MSI & & & & & 0.62 & 0.06 & & & & & 0.67 & 0.01 \\
\hline
\end{tabular}

Significant correlations $(p<0.05)$ shown in bold 

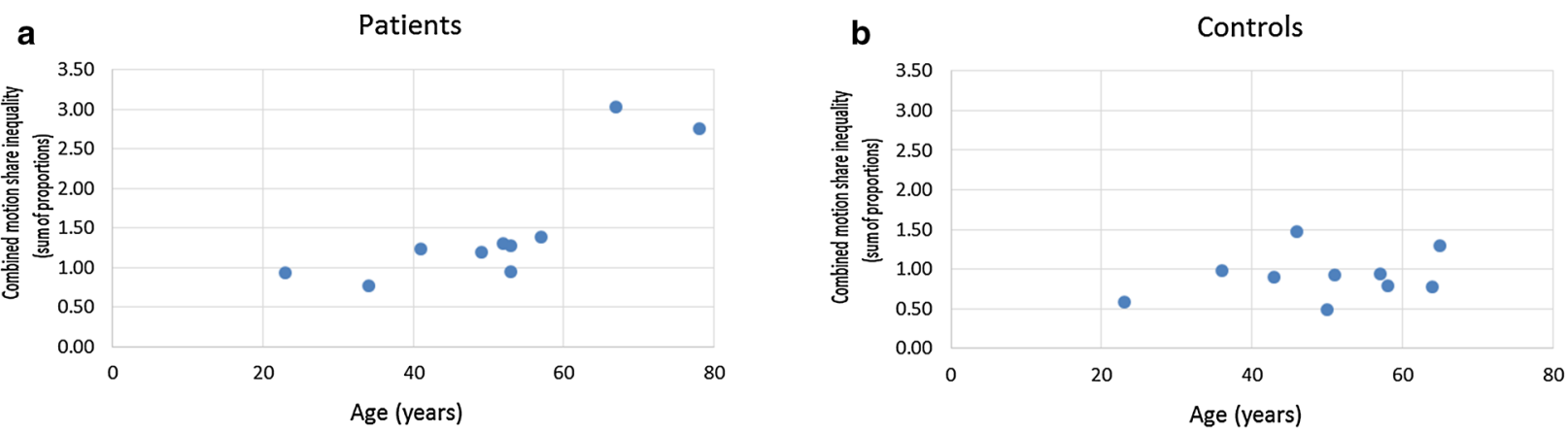

Patients
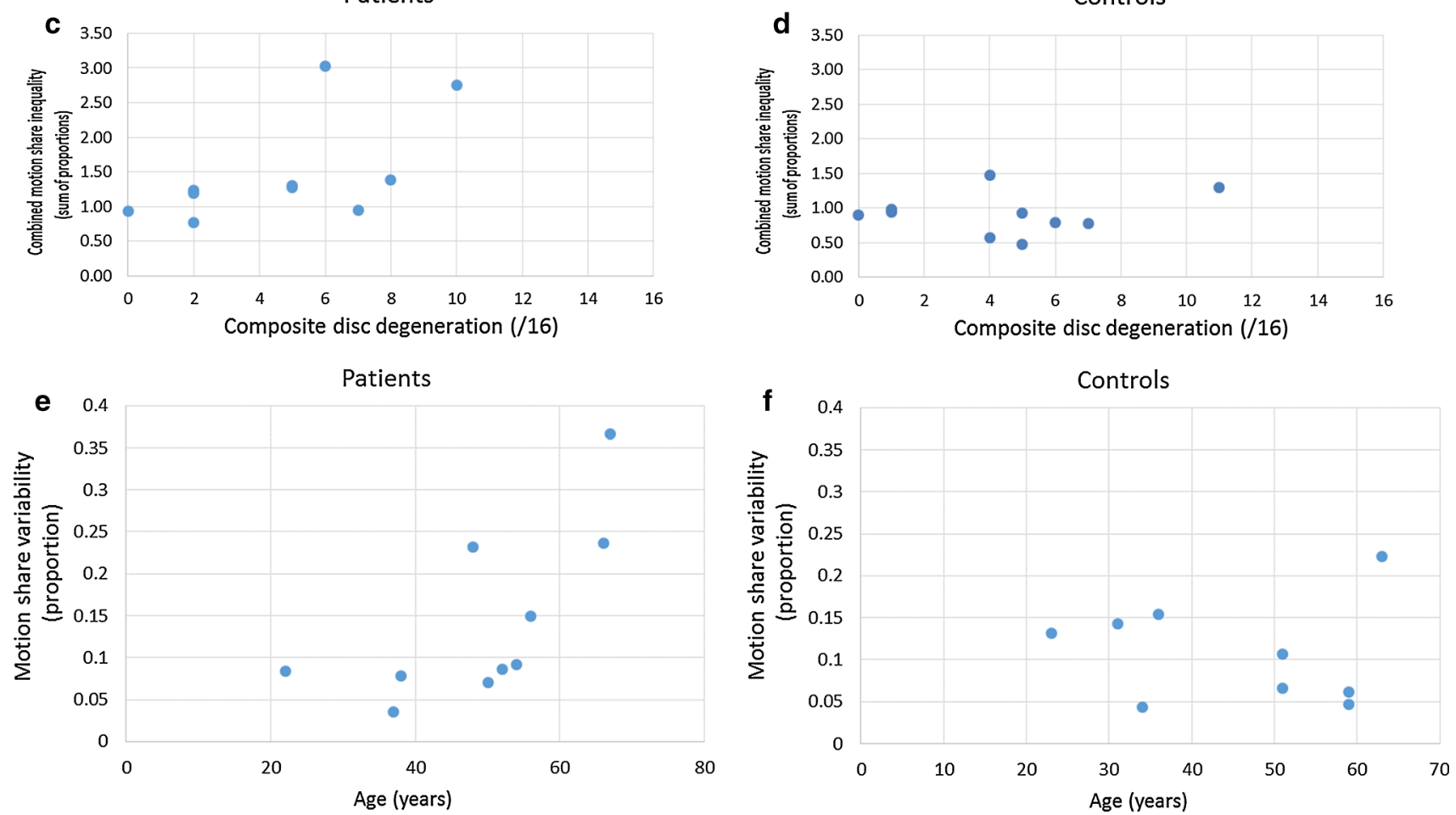

Patients

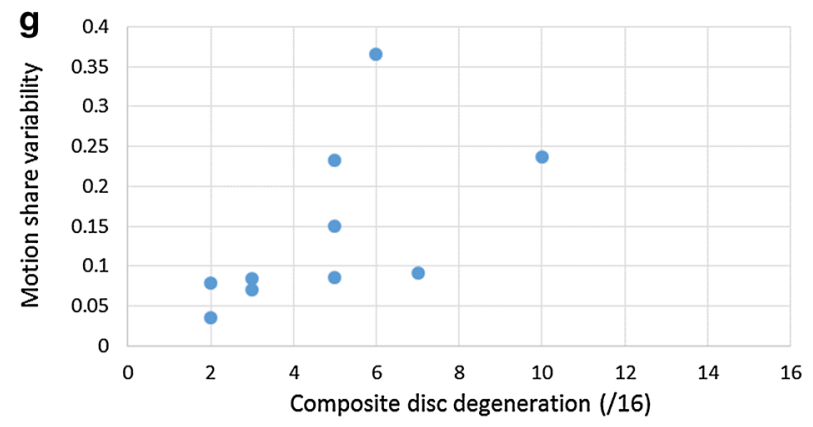

Fig. 4 Relationships between age, combined disc degeneration, MSI and MSV in patients and controls. Cohort 1. Age vs. MSI in combined recumbent flexion, extension, left and right motion. a Patients $R=0.85, p=0.004, \mathbf{b}$ controls $R=0.12, p=0.76$. Cohort 1 . Combined disc degeneration vs. MSI in combined recumbent flexion, extension, left and right motion. c Patients $R=0.70, p=0.03$,

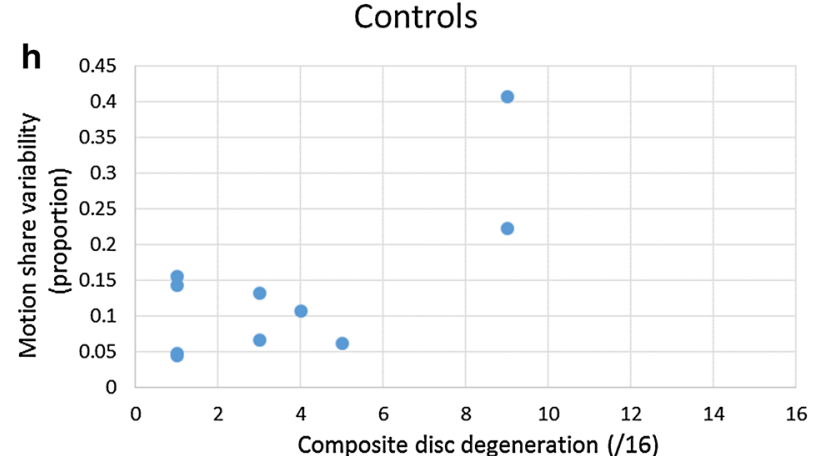

d controls $R=-0.15, p=0.67$. Cohort 2. Age vs. MSV in weight bearing flexion motion. e Patients $R=0.77, p=0.01$, f controls $R=0.25, p=0.49$. Cohort 2. Combined disc degeneration vs. MSV in weight bearing flexion motion, $\mathbf{g}$ patients $R=0.85, p=0.004$, h controls $R=0.47, p=0.18$ 
to disc degeneration when there is uneven restraint in the passive subsystem. Barz et al. put forward a new conceptual model of CNSLBP that links such structural degeneration with mechanical compensation and stability [38]. Thus, more evenly shared restraint (MSI), despite the presence of degenerative change, may be seen as the result of structural compensation that allows the individual relative freedom from symptoms.

In contrast, in weight bearing active flexion in patients, the correlations found between age, disc degeneration and increased variability of motion sharing (MSV) suggests relationships with control in the active subsystem. However, the finding that motion sharing variability (MSV) was not greater in patients during active weight bearing motion may have been due to the stabilising influence of the trunk muscles during active bending. Thus, control of MSV may be an important factor in the avoidance of CNSLBP. This possibility could be explored by future research using the above techniques in combination with electromyography [39]. However, Von Forell et al. found, using finite element modelling, stresses on the spinal holding elements would generally be lower when not all lumbar discs are degenerated [40].

It is uncertain whether these relationships are causative or consequent to back pain, or both. The above suggested relationships to rapid accelerations and/or increased muscle metabolic demands are possible explanations for a causative effect. For example, in the recumbent studies, it is difficult to conceive how pain alone could have selectively affected passive segmental restraint when muscle electrical activity was minimal [7].

Recent research by Lao et al. [41] supported the hypothesis of Kirkaldy-Willis [42] that disc degeneration has different effects on intervertebral motion at different stages. Contemporaneous discographic and profilometry studies have supported the hypothesis that painful discs are also usually disrupted [43]. The strong associations found here between disc degeneration and uneven intervertebral motion in patients, but not in controls, seem consistent with this. It is also consistent with other recent research, which found that disc degeneration was associated with low back pain, especially when associated with end-plate signal change [11, 44, 45]. However, unlike the present work, these studies used MRI disc degeneration grading, while radiographic grading based on structural aspects rather than biochemical changes may be better correlated with pain when considered alongside intervertebral motion patterns.

The other two main biological generators of CNSLBP are chemical and neuroplastic. Circulating inflammatory markers have been found in such patients [46] and it may be hypothesised that the greater the unevenness of motion sharing, the greater the likely prevalence of rapid displacements during physical tasks causing the release of cytokines from failing holding elements.
Central sensitisation seems to be a consequence of many factors that are linked to the experience of having chronic pain; however, the role of nociception, once it has become embedded, is less clear [47]. Exercise in the treatment of chronic pain has neurological as well as mechanical effects, making its monitoring by brain mapping a variable of interest for comparison with changes in the above mechanical factors with treatment [48]. Future work could, therefore, usefully compare MSI and MSV in passive recumbent and active weight bearing motion in patients and controls to monitor their responses to physical therapies.

\section{Limitations}

The main limitation of these studies is their small numbers. However, the strength and significance of the correlations and replication of previous work suggests that the relationships found should be robust. Many clinicians would prefer weight bearing motion studies to be conducted using free bending rather than with the pelvis constrained to capture 'natural' motion patterns. However, this makes comparison between participant groups difficult, as it introduces sources of extraneous uncontrolled variation, including that from large hip joint motions [26]. It would also have been useful to have obtained both recumbent and weight bearing sequences for both patients and controls in Cohort 2, but this was not possible owing to missing data and will need to wait for the future studies.

Lastly, imaging studies that use image intensifiers are associated with a radiation dose; however, image acquisition times for such studies are considerably less protracted than in clinical procedures, resulting in smaller doses. Mellor, reported a mean effective radiation dose of $0.561 \mathrm{mSv}$ for the four sequences which were used with Cohort 1 in the present studies. This is approximately half the dose of a conventional plain radiographic examination of the lumbar spine [49].

\section{Conclusion}

This research confirms and extends the results of previous studies [8, 24, 29] that found abnormalities of shared intervertebral motion to be consistent with having CNSLBP and suggests possible mechanisms for this. It also suggests routes for improved understanding of the role of disc degeneration in common back pain in which degeneration may be considered a pain source when it is associated with uneven motion sharing and end-plate signal change. The results open a route to the study of motion sharing as a moderator of outcomes and of prognosis in clinical studies and its role among other known biological 
factors, such as muscle metabolic demands and chemical markers. However, further confirmatory work is still needed.

Acknowledgements This project was funded by the European Council for Chiropractic Research Excellence.

\section{Compliance with ethical standards}

Conflict of interest The authors declare that they have no conflicts of interest.

Open Access This article is distributed under the terms of the Creative Commons Attribution 4.0 International License (http://crea tivecommons.org/licenses/by/4.0/), which permits unrestricted use, distribution, and reproduction in any medium, provided you give appropriate credit to the original author(s) and the source, provide a link to the Creative Commons license, and indicate if changes were made.

\section{References}

1. Leone A, Guglielmi G, Cassar-Pullicino VN, Bonomo L (2007) Lumbar intervertebral instability: a review. Radiology 245:62-77

2. Breen A, Dupac M, Osborne N (2016) Measuring intervertebral stability in vivo: exploring the criterion validity of the initial attainment rate. Orthop Proc 98-B:11

3. Wong K, Luk K, Leong J, Wong S, Wong K (2006) Continuous dynamic spinal motion analysis. Spine 31:414-419

4. Teyhen DS, Flynn TW, Childs JD, Abraham LD (2007) Arthrokinematics in a subgroup of patients likely to benefit from a lumbar stabilization exercise program. Phys Ther 87:313-325

5. Ahmadi A, Maroufi N, Behtash H, Zekavat H, Parnianour M (2009) Kinematic analysis of dynamic lumbar motion in patients with lumbar segmental instability using digital videofluoroscopy. Eur Spine J 18:1677-1685

6. Panjabi MM (2003) Clinical spinal instability and low back pain. J Electromyogr Kinesiol 13:371-379

7. Mellor F, Muggleton JM, Bagust J, Mason W, Thomas PW, Breen AC (2009) Mid-lumbar lateral flexion stability measured in healthy volunteers by in vivo fluoroscopy. Spine 34:E811-E817

8. Teyhen DS, Flynn TW, Childs JD, Kuklo TR, Rosner MK, Polly DW, Abraham LD (2007) Fluoroscopic video to identify aberrant lumbar motion. Spine 32:E220-E229

9. du Rose A, Breen A (2016) Relationships between lumbar intervertebral motion and lordosis in healthy adult males: a cross sectional cohort study. BMC Musculoskelet Disord 17:121

10. Breen AC, Dupac M, Osborne N (2015) Attainment rate as a surrogate indicator of the intervertebral neutral zone length in lateral bending: an in vitro proof of concept study. Chiropr Man Ther 23:28. doi:10.1186/s12998-015-0073-8

11. Teraguchi M, Yoshimura N, Hashizume H, Muraki S, Yamada H, Oka H, Minamide A, Nakagawa HM, Ishimoto Y, Nagata K, Kagotani R, Tanaka S, Kawaguchi H, Nakamura K, Akune T, Yoshida M (2015) The association of combination of disc degeneration, end plate signal change, and Schmorl node with low back pain in a large population study: the Wakayama Spine Study. Spine J 15:622-628

12. Brayda-Bruno M, Tibiletti M, Ito K, Fairbank J, Galbusera F, Zerbi A, Roberts S, Wachtel E, Merkher Y, Sivan SS (2014) Advances in the diagnosis of degenerated lumbar discs and the possible clinical application. Eur Spine J Suppl 3:s315-s323. doi:10.1007/800586-013-2960-9

13. Hemming R, Sheeran L, van Deursen R, Martin RW, Sparkes V (2015) Regional spinal kinematics during static postures and functional tasks in people with non-specific chronic low back pain. Int J Ther Rehabil 22:S8

14. D'hooge R, Hodges P, Tsao H, Hall L, MacDonald D, Danneels D (2013) Altered trunk muscle coordination during rapid trunk flexion in people in remission of recurrent low back pain. J Electromyogr Kinesiol 23:173-181

15. van Dieen JH, Cholewicki J, Radebold A (2003) Trunk muscle recruitment patterns in patients with low back pain enhance the stability of the lumbar spine. Spine 28:834-841

16. Shirazi-Adl A, El-Rich M, Pop DG, Parnianpour M (2005) Spinal muscle forces, internal loads and stability in standing under various postures and loads-application of kinematics-based algorithm. Eur Spine J 14:381-392. doi:10.1007/s00586-0040779-0

17. Goel VK, Goyal S, Clark C, Nishiyama K, Nye T (1985) Kinematics of the whole lumbar spine: effect of discectomy. Spine 10:543-564

18. Schultz AB, Warwick DN, Berkson MH, Nachemson AL (1979) Mechanical properties of human lumbar spine motion segments-Part 1: responses inflexion, extension, lateral bending and torsion. J Biomech Eng 101:46-52

19. Tencer AF, Ahmed AM, Burke DL (1982) Some static mechanical properties of the lumbar intervertebral joint, intact and injured. J Biomech Eng 104:193-201

20. Li G, Wang S, Passias P, Xia Q, Li G, Wood K (2009) Segmental in vivo vertebral motion during function human lumbar spine activities. Eur Spine J 18:1013-1021

21. Passias PG, Wang S, Kozanek M, Xia Q, Li W, Grottkau B, Wood KB, Li G (2011) Segmental lumbar rotation in patients with discogenic low back pain during functional weight-bearing activities. J Bone Jt Surg Am 93:29-37

22. Pearcy M, Portek I, Shepherd J (1985) The effect of low back pain on lumbar spinal movements measured by three-dimensional X-ray analysis. Spine 10:150-153

23. Plamondon A, Gagnon M, Maurais G (1988) Application of a stereoradiographic method for the study of intervertebral motion. Spine 13:1027-1032

24. Abbott J, Fritz J, McCane B, Shultz B, Herbison P, Lyons B, Stefanko G, Walsh R (2006) Lumbar segmental mobility disorders: comparison of two methods of defining abnormal displacement kinematics in a cohort of patients with non-specific mechanical low back pain. BMC Musculoskelet Disord 7:45

25. Kanayama M, Abumi K, Kaneda K, Tadano S, Ukai T (1996) Phase lag of the intersegmental motion in flexion-extension of the lumbar and lumbosacral spine: an in vivo study. Spine 21:1416-1422

26. Harada M, Abumi K, Ito M, Kaneda K (2000) Cineradiographic motion analysis of normal lumbar spine during forward and backward flexion. Spine 25:1932-1937

27. Okawa A, Shiomiya K, Komori H, Muneta T, Arai Y, Nakai O (1998) Dynamic motion study of the whole lumbar spine by videofluoroscopy. Spine 23:1743-1749

28. Wong KWN, Leong JCY, Chan M-K, Lu WW (2004) The flexion-extension profile of lumbar spine in 100 healthy volunteers. Spine 29:1636-1641

29. Mellor FE, Thomas P, Thompson P, Breen AC (2014) Proportional lumbar spine inter-vertebral motion patterns: a comparison of patients with chronic non-specific low back pain and healthy controls. Eur Spine J 23:2059-2067. doi:10.1007/s00586-014-3273-3

30. Aiyangar A, Zheng L, Anderst W, Zhang X (2015) Apportionment of lumbar L2-S1 rotation across individual motion segments during a dynamic lifting task. J Biomech 48:3709-3715 
31. Reeves NP, Narendra KS, Cholewicki J (2007) Spine stability: the six blind men and the elephant. Clin Biomech 22:266-274

32. Breen A, Muggleton J, Mellor F (2006) An objective spinal motion imaging assessment (OSMIA): reliability, accuracy and exposure data. BMC Musculoskelet Disord 7:1-10

33. Kellgren JH, Lawrence JS (1958) Osteo-arthrosis and disc degeneration in an urban population. Ann Rheum Dis 17:388-397

34. Shiba Y, Taneichi H, Inami S, Moridaira H, Takeuchi D, Nohara Y (2016) Dynamic global sagittal alignment evaluated by threedimensional gait analysis in patients with degenerative lumbar kyphoscoliosis. Eur Spine J 25:2572-2579

35. D'hooge R, Cagnie B, Crombez G, Vanderstraeten G, Achten E, Danneels L (2013) Lumbar muscle dysfunction during remission of unilateral recurrent nonspecific low-back pain: evaluation with muscle functional MRI. Clin J Pain 29:187-194

36. Panjabi MM (2006) A hypothesis of chronic back pain: ligament subfailure injuries lead to muscle control dysfunction. Eur Spine J 15:668-676

37. Zhao F, Pollintine P, Hole BD, Dolan P, Adams MA (2005) Discogenic origins of spinal instability. Spine 30:2621-2630

38. Barz T, Melloh M, Lord SJ, Kasch R, Merk HR, Staub LP (2014) A conceptual model of compensation/decompensation in lumbar segmental instability. Med Hypotheses 83:312-316

39. du Rose A, Breen A (2016) Relationships between paraspinal muscle activity and lumbar inter-vertebral range of motion. Healthcare. doi:10.3390/healthcare4010004

40. Von Forell GA, Stephens TK, Samartzis D, Bowden AE (2015) Low back pain: a biomechanical rationale based on "patterns" of disc degeneration. Spine 40:1165-1172

41. Lao L, Daubs MD, Scott TP, Lord EL, Cohen JR, Tin R, Zhong G, Wang JC (2015) Effect of disc degeneration on lumbar segmental mobility analyzed by kinetic magnetic resonance imaging. Spine 40:316-322
42. Kirkaldy-Willis WH (1992) Pathology and pathogenesis of low back pain. In: Kirkaldy-Willis WH, Burton CV (eds) Managing low back pain. Churchill Livingstone, New York, pp 49-79

43. McNally DS, Shackleford IM, Goodship AE, Mulholland RC (1996) In vivo stress measurement can predict pain on discography. Spine 21:2580-2587

44. Takatalo J, Karppinen J, Niinimaki J, Taimela S, Nayha S, Mutanen P, Sequeiros RB, Kyllonen E, Tervonen O (2011) Does lumbar disc degeneration on magnetic resonance imaging associate with low back symptom severity in young Finnish adults. Spine 36:2180-2189

45. Maatta JH, Wadge S, MacGregor A, Karppinen J, Williams FM (2015) ISSLS prize winner: vertebral endplate (modic) change is an independent risk factor for episodes of severe and disabling low back pain. Spine 40:1187-1193

46. Li Y, Liu J, Liu Z-Z, Duan D-P (2016) Inflammation in low back pain may be detected from the peripheral blood: suggestions for biomarker. Biosci Rep 36:e0361

47. Nijs J, Van Houdenhove B, Oostendorp RAB (2010) Recognition of central sensitization in patients with musculoskeletal pain: application of pain neurophysiology in manual therapy practice. Man Ther 15:135-141

48. Pelletier R, Higgins J, Bourbonnais D (2015) Is neuroplasticity in the central nervous system the missing link to our understanding of chronic musculoskeletal disorders? BMC Musculoskelet Disord. doi:10.1186/s12891-015-0480-y

49. Mellor FE, Thomas P, Breen A (2014) Moving back: the radiation dose received from lumbar spine quantitative fluoroscopy compared to lumbar spine radiographs with suggestions for dose reduction. Radiography 20:251-257 